\title{
A STUDY OF THE TURBULENT FLOWS BEHIND A RECTANGULAR SHARP CRESTED WEIR IN OPEN CHANNEL USING LASER DOPPLER VELOCIMETRY
}

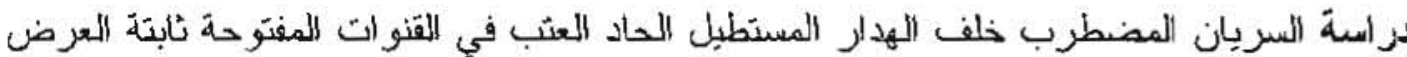 \\ وذلاك بإستخدام جهاز اللميزر الحديث \\ M.I. ATTIA
}

Associ. Prof. Water \& Water Structures Engg. Dept., Faculty of Engg., Zagazig University, Zagazig, Egypt

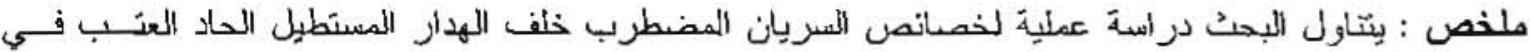

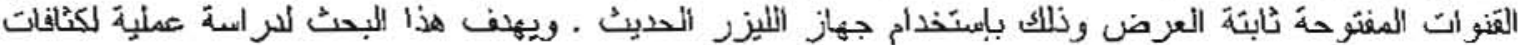
الإضطر اب و السر عات المتوسطة لاتجاه اللسريان و الاتجاه الر أستي (Depthwise) عند تطاعات عرضية مختلفة

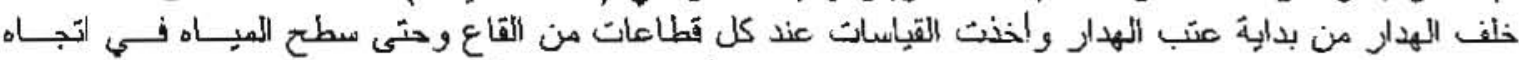

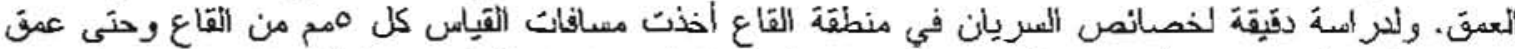

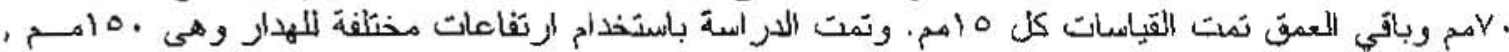

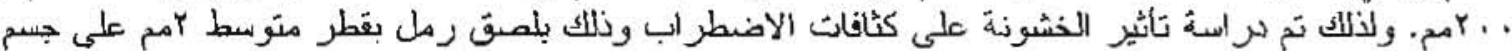

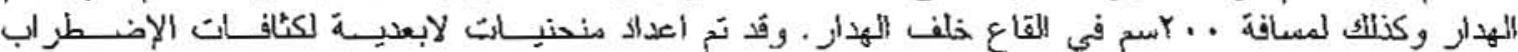

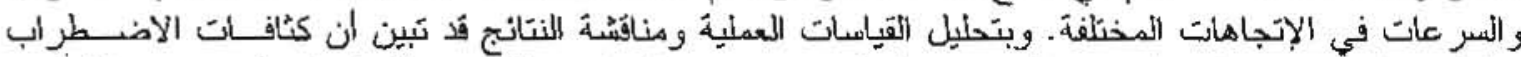

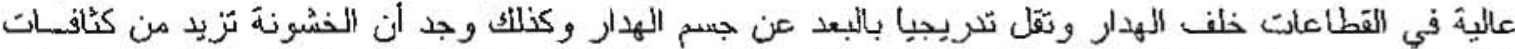

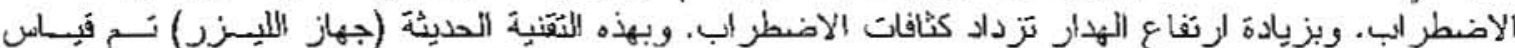

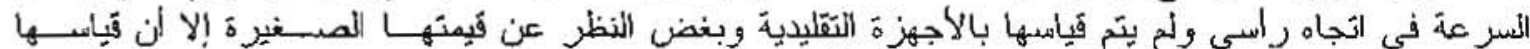

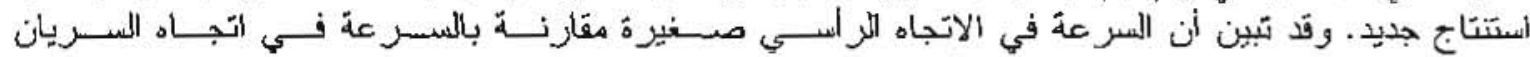
(Stremwise) عدةَ تَطاعات مختلفة خلف الهدار .

\section{ABSTRACT:}

This paper describes the experimental investigation using laser Doppler velocimetry (LDV) in the downstream of the rectangular sharp crested weir in a horizontal rectangular channel of constant width. The study was carried out for flows on smooth and rough weir. For precise and accurate measurements of mean and fluctuating flow characteristics such as streamwise and vertical turbulence intensity components and streamwise and vertical mean velocity components. The depthwise measurements were carried out for different heights of the weir along the centerline at different cross sections downstream of weir in the wake region. The results show that, the measured values of turbulence intensities are found to have high level of turbulence in the near wake region but are low in the far wake region. The turbulence intensities depict the occurrence of a constant turbulence close to the wall in the far wake region. The roughness was found to increase the turbulence. Also, it can be seen that with increasing weir height, the turbulence intensities are increased.

1- INTRODUCTION:

Flow fields associated with separation and reattachment have received significant attention because of their importance in many engineering applications. Examples include the flow behind the back ward-facing step, separated flow in diffusers, and separation bubbles on airfoils. Among these separation reattachment configuration, the flow behind rectangular sharp crested weir. To study the physics of flow separation behind the rectangular sharp crested weir, the simple geometry and the easily attainable two dimensionality of the test flow facilitate the analysis of separation induced flow 
C. 2 M. I. Attia

phenomena, i.e. the determination of mean and fluctuating flow quantities such as streamwise and vertical components of turbulence intensity, and streamwise and vertical mean velocity components. A detailed review of the two-dimensional situations has been offered by Bradshaw and Wong [4] and later by Eaton and Johnston [7]. The reattachment Iength, one of the important properties because it indicates the rate of mixing of the separated shear layer, has been found by Eaton and Johnston $[7]$ to be sensitive to many parameters, e.g., ReynoIds number, background turbulence level, streamwise pressure gradient, etc. Near the reattachment region, the local turbulence intensity and Reynolds stresses reach thcir peak values, which can be attributed to the impingement of the unsteady shear layer on to step's floor. The coherent structures on the shear layer were studied recently by Bhattacharjee et al. [3] and Roos and Kegelman [12]. In a two-dimensional backward facing step, the instantaneous velocity traces indicate that the coherent structures in the shear layer are correlated almost across the entire span, Bhattacharrjee et al. [3]. Flow visualization by Cherdron et al. [5] showed vortex pair structures behind a sudden expansion inside a symmetric duct. In a channel with a fully developed velocity profile before the step, Armaly et al. [1] found multiple regions of separation downstream of the backward facing step on both the top and bottom sides of the channel walls. Their measurements showed that the appearance of 2- EXPERIMENTAL SET UP AND

\section{TEST PROCEDURE}

The measurements were carried out in a horizontal rectangular open channel that is $9500 \mathrm{~mm}$ long, $300 \mathrm{~mm}$ width and 500 $\mathrm{mm}$ height with glass wall $6 \mathrm{~mm}$ thick and a stecl plate bed. Figure 1 depicts layout of the test facility. The water is supplied from a a separation bubble on the wall opposite to the step destroyed the two-dimensionally of the flow, and wavy patterns of the spanwise separation-reattachment locations existed for both top and bottom separation bubbles. Their numerical results supported the existence of those additional separation regions. Several recent Navier-Stock computational work, Kim ad Monin [9] and Kaiktsis et al. [8|. A great number of detail studies, Ruck et al. [13], Nakagawa et al. [11], Ethcidge et al. [6], and Amano et al. [2] have been published in the past on backward facing single sided step flows which describc the interactions of limiting geometrical parameters and flow characterizing quantities mostly in a time averaged version. Nashat [16] presented the model of vortex shedding for steady separated flow over a normal wall. Sinulation of turbulent flow separation through closed rectangular conduit has bcen pointed by Nashat et al.[14].

The flow characteristics after a downward facing step in channel bed have been reported by Nashat al. [15]. The aim of the present research depicts the results of laser Doppler Velocimetry (LDV) investigation behind of rectangular sharp crested weir in a horizontal rectangular channel of constant width. Experiments are carried out on smooth and rough weir to study the turbulence intensity components, streamwise and vertical mean velocity and the effect of the roughness on the turbulence intensitics.

constant head overlicad tank to the fume at a desired discharge that is continuously monitored with an on-line orifice meter. The flume side walls are made up of $6 \mathrm{~mm}$ thick glass sheets. A tail vertical gate is provided at the downstream end of the flume to maintain 
a required water depth of channcl flow. The water is finally collected in a sump placed in the basement from where it is pumped back to the overhead tank by a $16 \mathrm{HP}$ pump.

With reference to the origin fixed at the bed along the centerline, transverse of measuring volume was run to obtain the profiles of both the mean vclocity components and RMS of turbulence intensities. The measuring points were closely spaced in the region of high velocity gradient. All the measurements were made for a constant discharge rate of $40 \mathrm{~V} / \mathrm{s}$ on the free stream water depth of $320 \mathrm{~mm}$. This gave Reynolds number based on the frec stream velocity $4 \times 10^{4}$ which ensured the turbulent flow for all the test conditions. Froude number of the free stream flow $\mathrm{Fr}=$ 0.230 , ensured the free stream flow to bc subcritical. To obtain the vertical profiles of the mean and fluctuating quantities, the measurements were conducted in the vertical plane along the centerline at different locations downstream the rectangular sharp crested weir. In the vertical direction along the depth, 30 measurements at $5 \mathrm{~mm}$ intervals up to $65 \mathrm{~mm}$ from the bed boundary and $15 \mathrm{~mm}$ for the rest were taken.

\section{3-INSTRUMENTATION}

The experimental data were collected using a DANTEC LDV system, consisted of a 5 watt-ion laser with two laser beams one blue $(488 \mathrm{~nm})$ and one green $(514.5 \mathrm{~nm})$, a Fiber-optic measuring probe in back-scater mode, two Burst Spectrum Analyzer (BSA) were used to evaluate the Doppler frequencies, and subsequent computer analysis consisted to velocity bias averaging and outlier rejection. Figure 2 shows a block diagram of the two component LDV set up used for the measurements. On a traverse bench, the measuring probe (laser beams or measuring volume) was focused at a measuring point from one side of the channcl glass wall through an optical lens. The number of samples taken at every point was 5000 bursts. This correspond to a sample averaging time of about 100 seconds. The data rate was about 10-20 $\mathrm{HZ}$. Before acquiring the data, the LDV signal was checked for its regular Doppler burst that correspond to a particle passing through the measuring volumc. The measurements werc taken at different positions downstream of the rectangular sharp crested weir for $Q=40$ $1 / s$. Figure 3 shows the location grid of the measuring sections $(x / H)$ downstream the weir. The weir was fabricated from transparent prespex sheets, that is $260 \mathrm{~mm}$ and width $20 \mathrm{~mm}$ thick. The height (H) of the weir was taken $150 \mathrm{~mm}$ and $200 \mathrm{~mm}$.

\section{3-RESULTS AND DISCUSSION:}

(a) Streamvise Mean Velocity Disfribution (II) I $\left.\underline{\mathrm{U}}_{0}\right)$ along the Depth:

The values of u-component of streamwise mean velocity, made nondimensional with respect to the streamwise frec stream velocity $U_{0}$. Figure 5 depicts the profiles of streamwise mean velocity component $\overline{\mathrm{i}} / \mathrm{U}_{0}$ behind the rectangular sharp crested weir along the depth at different locations. The profiles of $u / U_{0}$ along the longitudinal direction at the centerline exhibit the expected trends of flow separation, shear layer growth, and reattachment (reattachment occurs at $\mathrm{x} / \mathrm{H}=$ $8 \pm 0.3$ ). Dircetly downstream the w.ir, reversed flow and flow separation could be observed downstream the weir as shown in Fig. 5 at $\mathrm{x} / \mathrm{H}=1.0$ as can be seen by the shapc of the velocity profile and was observed by dye injection. Thesc observation are consistent with the backstep flow measurement of Ruck and Mokida (1990). 
(b) Vertical Mean Velncity Distribution $\left(\bar{v} / \mathrm{U}_{0}\right)$ ? along the Depth

The values of $\bar{v}$ - component of vertical mean velocity, made mon- dimensional with respect to the streamwise free stream velocity $\mathrm{U}_{0}$. Figure 6 depicts the profiles of dimensionless vertical mean velocity $\bar{v} / U_{o}$ behind the rectangular sharp crested along the depth at different locations at which streamwise mean velocities were measured. Although $\bar{v} / U_{0}$ fluctuates as one moves downstream of the weir, the magnitude decreases reaching relatively small value again at the farthest downstream of $x / H=10$. The zero magnitude of vertical component $\bar{v}$ / $\mathrm{U}_{0}$, occurs at more than onc point at several locations. This observation is somewhat more intriguing as one may not expect more than one location at which $\bar{v} / U_{0}$ could be zero. One may attribute the multiplicity of null point to the three dimensional interaction between the entrance flow to the weir, almost with negative vertical velocity component, the influence of side wall weir itself along with horizontal bed impeding the downward component of velocity. This complex interaction would influence the flow pattern giving rise to multiplicity of null point.

\section{(c) Streamvise Turbulence Intensities $u \% \mathrm{U}_{0}$} along the Depth:

Measurements were made for the two components of the fluctuating turbulence velocity ( $u^{\prime}$ and $v^{\prime}$ ) in terms of their root mean square (rms) values. However, bccause of the limitation of space, results for rms value of only the longitudinal component of turbulence fluctuations ( $u^{\prime}$ ) are presented for most cases. Root mean square (rms) of turbulence intensity made non-dimensional with respect to the frce stream velocity $\mathbf{U}_{0}$. Figure 7 depicts the profiles of streamwise component of dimensionless turbulence intensities $u \% U_{n}$ with relative water depth $y /$ $y_{0}$ for different weir lieight of 150 and 200 $\mathrm{mm}$ at different locations behind the rectangular sharp crested weir for discharge $40 \mathrm{l} / \mathrm{s}$. As a comprehensive observation, it is noted that the nature of distribution of the II/ $U_{0}$ component of turbulence intensity is essentially the same for both heights. The turbulence $u^{\circ} / \mathrm{U}_{0}$ of the weir height $200 \mathrm{~mm}$ is always stronger compared to the turbulence of the weir height $150 \mathrm{~mm}$. A high level of turbulence in the near wake region $(x / H=1$ and 2$)$ results from the disturbance to the flow caused by the rectangular sharp crested weir, with the increasing distance from the boundary, the turbulence intensity $1 \%$ / $U_{0}$ increase in wall region defined by $y / y_{0} \leq 0.2$ tcnding towards a maximum in the intermediate region (core region) defined by $0.2 \leq y^{\prime} y_{0} \leq 0.6$, turbulence intensity $u$ / $\mathrm{U}_{0}$ decease gradually in the upper region (free surface region) defined by $y / y_{0}>0.6$, reaching the minimum at the free surface. Since turbulence intensity gradually decrease as we move towards the wall, instead of attaining its maximum value. At $x / H=5$, the curve is more flattened and the $u / U_{0}$ component is partically constant in the central region (core region) of the flow. The maxima occurs very near the wall, indicating that the turbulence structure in the wall region is fully established. Also, Figure 8 depicts the variation of $11 \% \mathrm{U}_{0}$ with $y / y_{0}$ for rough and smooth weir. The roughness of the weir can be secn to cause higher level of turbulence throughout the flow region. Gencrally, the location of the minimum value of the turbulence intensity $u$ '/ $U_{0}$ occurs at the frec surface of the smooth and rough weir at all the cross sections. The bchaviour of the vertical turbulence intensity component $v / U_{0}$ was found to be essentially the same as that of the 
$\mathrm{u} / \mathrm{U}_{0}$ component, except that its magnitude was sinaller than that for the longitudinal 5. CONCLUSIONS:

The experimental study on the turbulent flows behind a rectangular sharp crested weir in open channel indicates that:

The turbulence intensities have a high level of turbulence in the near-wake rcgion, In the far-wake region the intensities are smaller and the profiles are similar in accordance with the fully developed flow. The roughness of the weir increases the intensity of turbulence. As a comprehensive obscrvation, it is noted that, the minima of turbulence intensities being located in the upper region (free surface region) defined by $y / y_{0}>0.6$, approximately at the free surface. In the near wake region at $\mathrm{x} / \mathrm{H}<3.5$ a high level of turbulence results from the disturbance to the flow caused by the rveir, maximum of turbulence occur in the core region (intermediate region) defined by $0.2 \leq$ $y / y_{0} \leq 0.6$. At $x / H>4.0$, the curve is move flattencd, the maxima occurs very near the wall indicating that the turbulence structure in the wall region is fully established. The component $u^{`} / \mathrm{U}_{0}$.

vertical mean velocity component $\bar{v} / U_{0}$ is observed to be small compared to the streamwise mean velocity $\overline{\mathrm{u}} / \mathrm{U}_{0}$. Regardless of its magnitude, measurement of the vertical velocity is a new finding using LDV tcchnique. Although $\bar{v} / U_{0}$ fluctuates as onc moves dowustream of the weir, the magnitude gradually decreases reaching small value again at the farthest downstream. The depthwise variation of $\tilde{\mathrm{u}} /$ $\mathrm{U}_{0}$ component and the $\bar{v} / \mathrm{U}_{0}$ component differ markedly, $\overline{\mathbf{v}} / \mathrm{U}_{0}$ may have multiple null points and positive as ivell as negative values. But $\overline{\mathbf{u}} / \mathrm{U}_{\mathrm{o}}$ predominantly positive all aTong the depth except at the location nearer the wake region where it can assume ncgative or a zero value along the vertical axis. The general features of the afore described observations are consistent with those for backstep flow's (Ruck and Mokiola, 1990) and (Nakagawa and Nezu, 1987).

\section{NOMENCLATURE:}

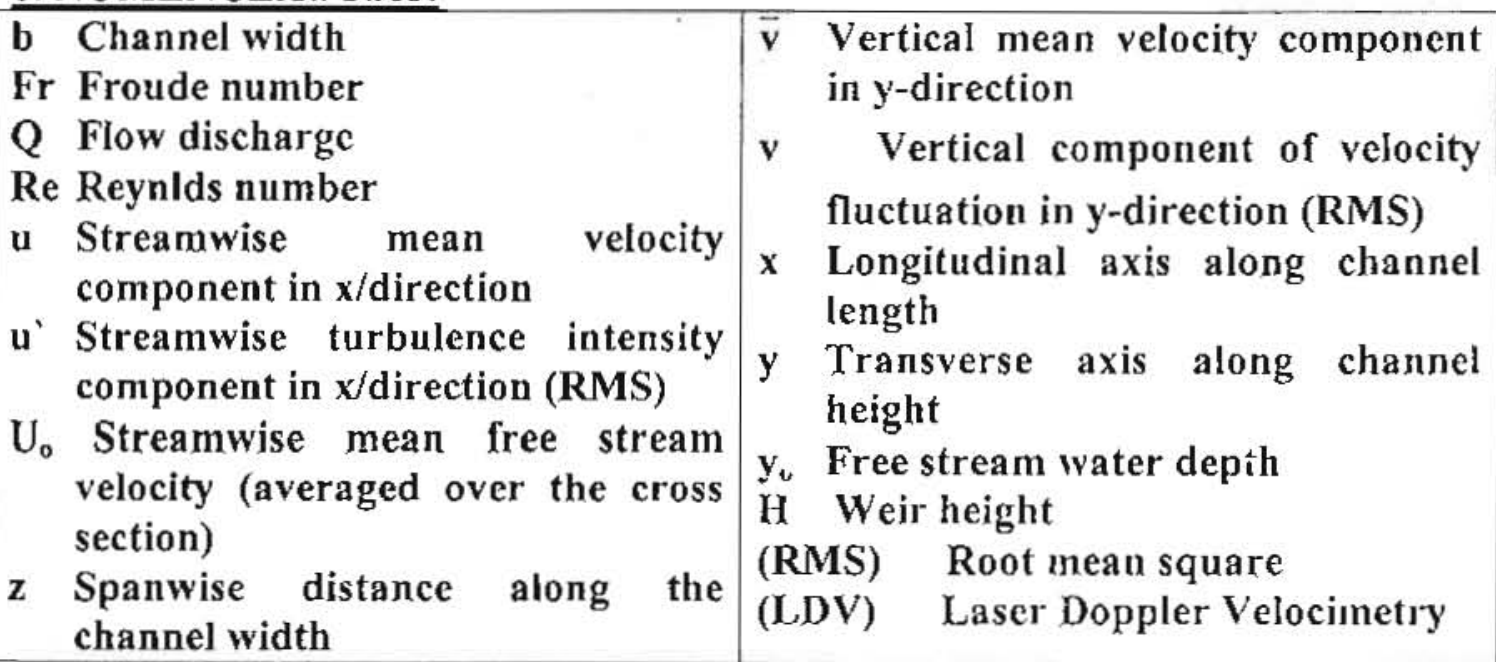




\section{REFERENCES:}

I- Armaly, B.F., and Durst, F., (1983), "Experimental and Theoretical Investigation of Backward Facing Step," J. of Fluid Mechanies, Vol. 127, pp. 473-496.

2-Amino, R.S., and Goel, P., (1985), "Computations of Turbulent Flow Beyond Backward Facing Stcps Using Reynolds Stress Closure," AIAA J., Vol. 23, No. 9, pp. 13561361.

3- Bhattacharjee, S., and Scheelke, B., (1986), Modification of Vortex Interactions in a Reattacting Flow," AIAA J., 24, No. 4, pp. 623-629.

4- Bradshaw, P., and Wong, F.Y., (1972), "The Reattachment and Relaxation of a Turbulent Shear Layer", J. Fluid Mech., Vol., 52, Part 1, pp. 113-135.

5- Chardron, W., and Durst, F., (1978), "Asymmetric Flows and Instabilities in Symmetric Ducts with Sudden Expansions", J. Fluid Mech., Vol. 84, pp. 13-31.

6- Etheridge, D.W., and Kemp, P.H., (1978), "Measurements of Turbulent Flow Downstream of a Rearward Facing Step", Fluid Mech., No. 3.

7- Eaton, J.K., and Johnston, J.P., "A Review of Research on Subsonic Turbulent Flow Reattachment, "AIAA J., Vol. 9, pp. 1093-1100.

8- Kaiktesis, L., and Orzag, S., (1991), "Onset of Three-Dimensionality, Equilibria, and Early Transition in Flow Over a Backward Facing Step," J. Fluid Mech., Vol. 231, pp. 501-528.

9- Kim, J., and Moin, P., (1985), "Application of a Fractional Step Method to Incompressible Navier-
Stokes Equations", J. Computational Physics, Vol. 59, pp. 308-323.

10-Nezu, I., and Nakagawa, (199.3), "Turbulence in Open Channel Flows "IAHR-Monograph, A, A, Balkema Publisers, Old Post Road, Bookficld, VT 05036, USA.

11-Nakagawa, II., and Ncz.II, I., (1987), "Expeimental Investigation on Turbulent Structure of Backward Facing Step Flow in an Open Channel, J. of Hydraulic Research, vol. 25, No. I, pp. 67-89.

12-Roos, F.W., and Kegelman, J.T., (1986) "Influence of Excitation on Coherent Structures in Reattaching Turbulent Shear Layers," AIAA paper No. 86-0112.

13-Ruck, B., and Mokida, (1990), "Flow over a Single Sided Backward Facing Step with Step Angle Variations," Proc. $3^{\text {rd }}$ Int Conf. of Laser Anemometry, BHRA, SpringierVerlag, UK. Pp. 369-378.

14- Nashat, A.A, Mohamed, D., and Ashraf, F., (2000), "Simulation of Turbulent flow Separation through closed Rectangular conduit with Onesided Abrupt Enlargement", Fifth Int. Water technology Conf., Alexandria, Wgypt.

15-Nashat, A.., (1995), " On the flow characteristics after a Downword facing Step in Channel Bed", Bulletin of the faculty of Engy., Assuit Univ., Vol.23, No.1, January, Egypt.

16- Nashat, A., A., (1991), In invescid Model of Vorter shedding for steady separated flow over a normal Wall", Bulletin of the faculty of Engg., Assuit Univ., Vol.19, No.,1, January, Egypt. 


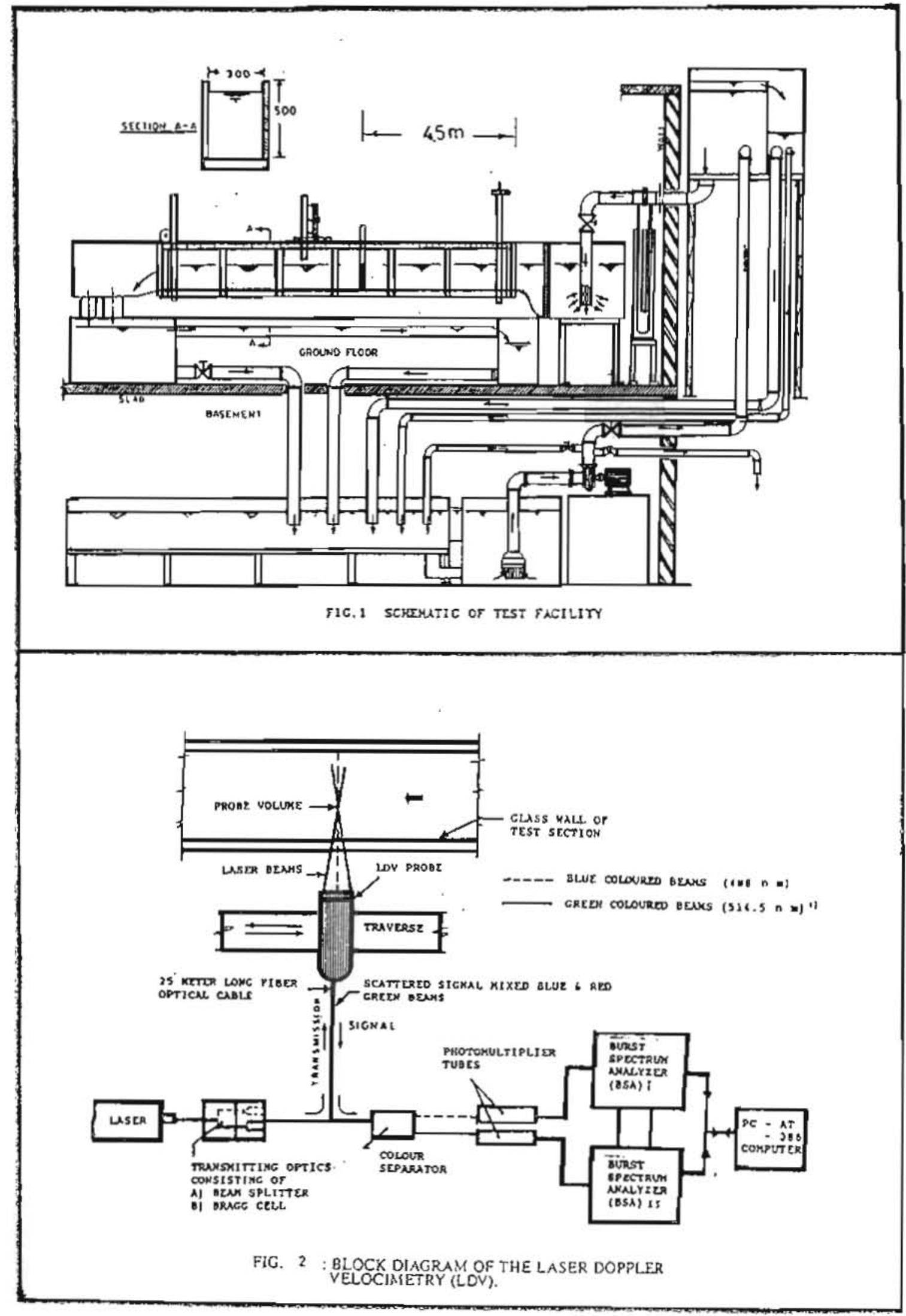




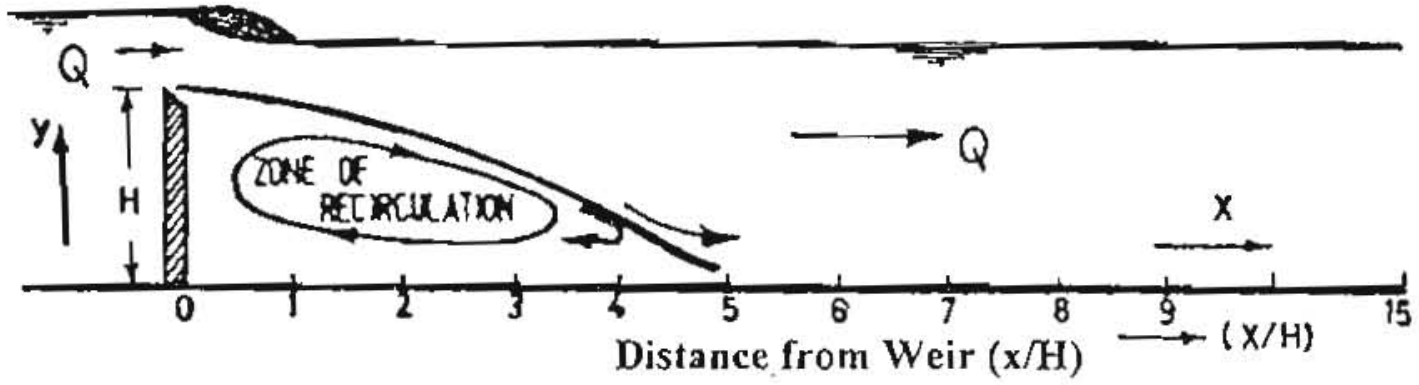

Fig. (3) Definition sketch of the rectangular sharp crested weir.

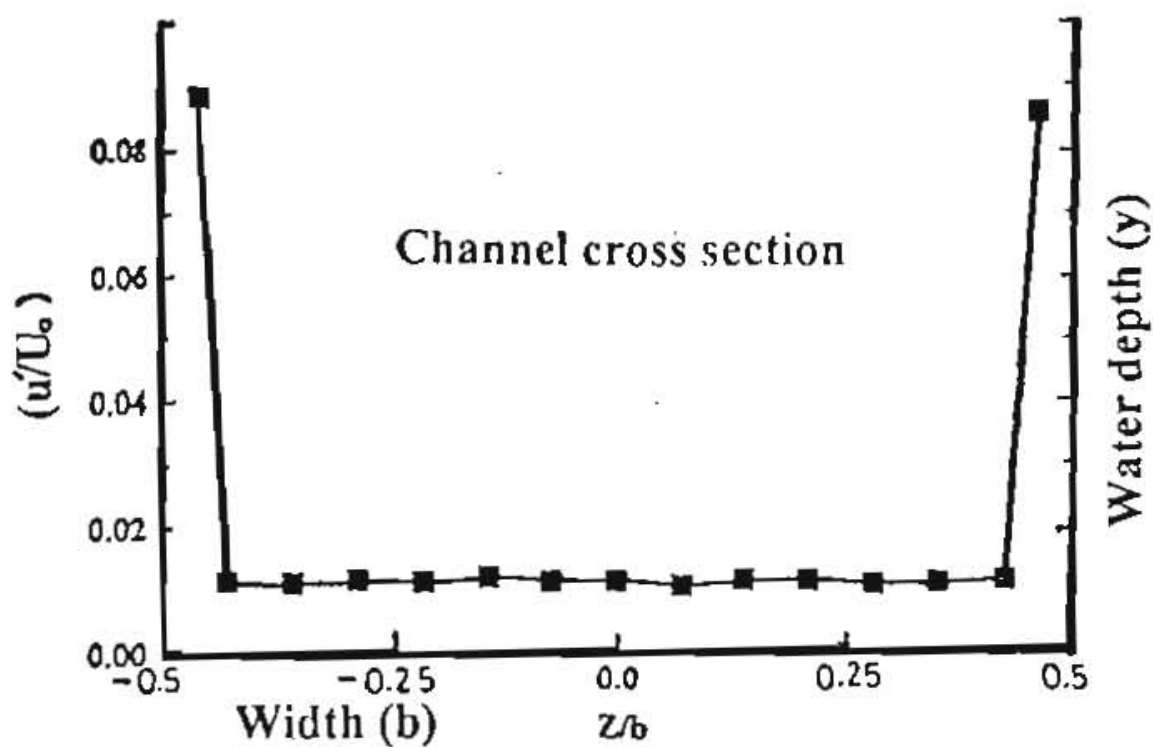

(b)Free streamwise turbulence intensity ( $u$ ' $\left.\mathrm{U}_{0}\right)$

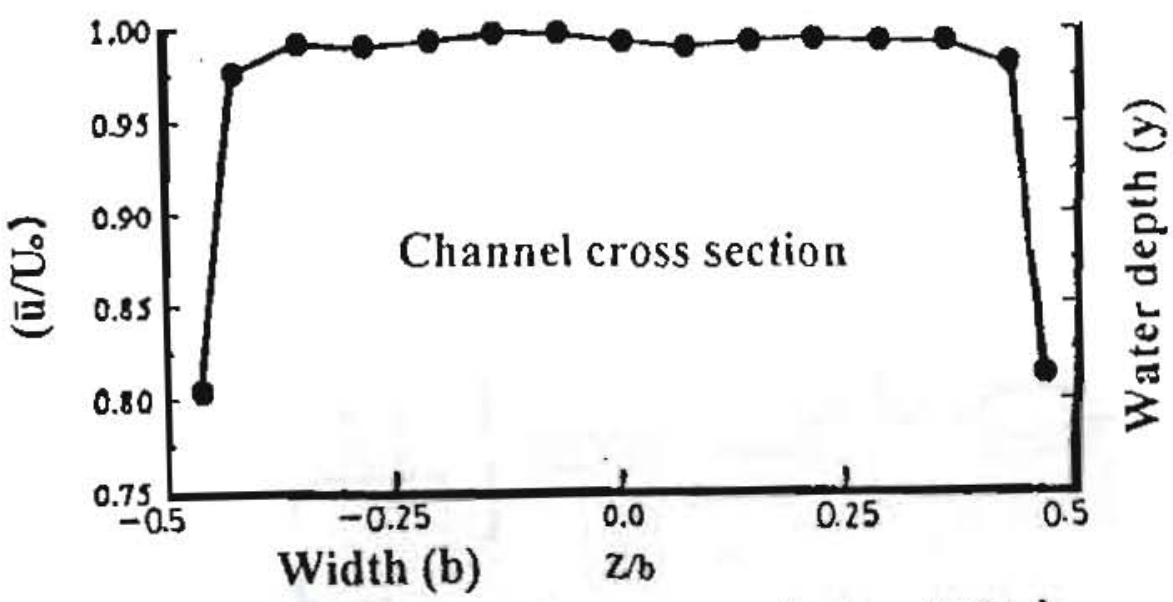

(a) Free streamwise mean velocity $\left(\bar{u} / U_{0}\right)$

Fig. (4) Spanwise profiles of the free stream velocity and turbulence intensity. 
Mansoura Engineering Joumal, (MEJ), Vol. 33, No. 3, September 2008.
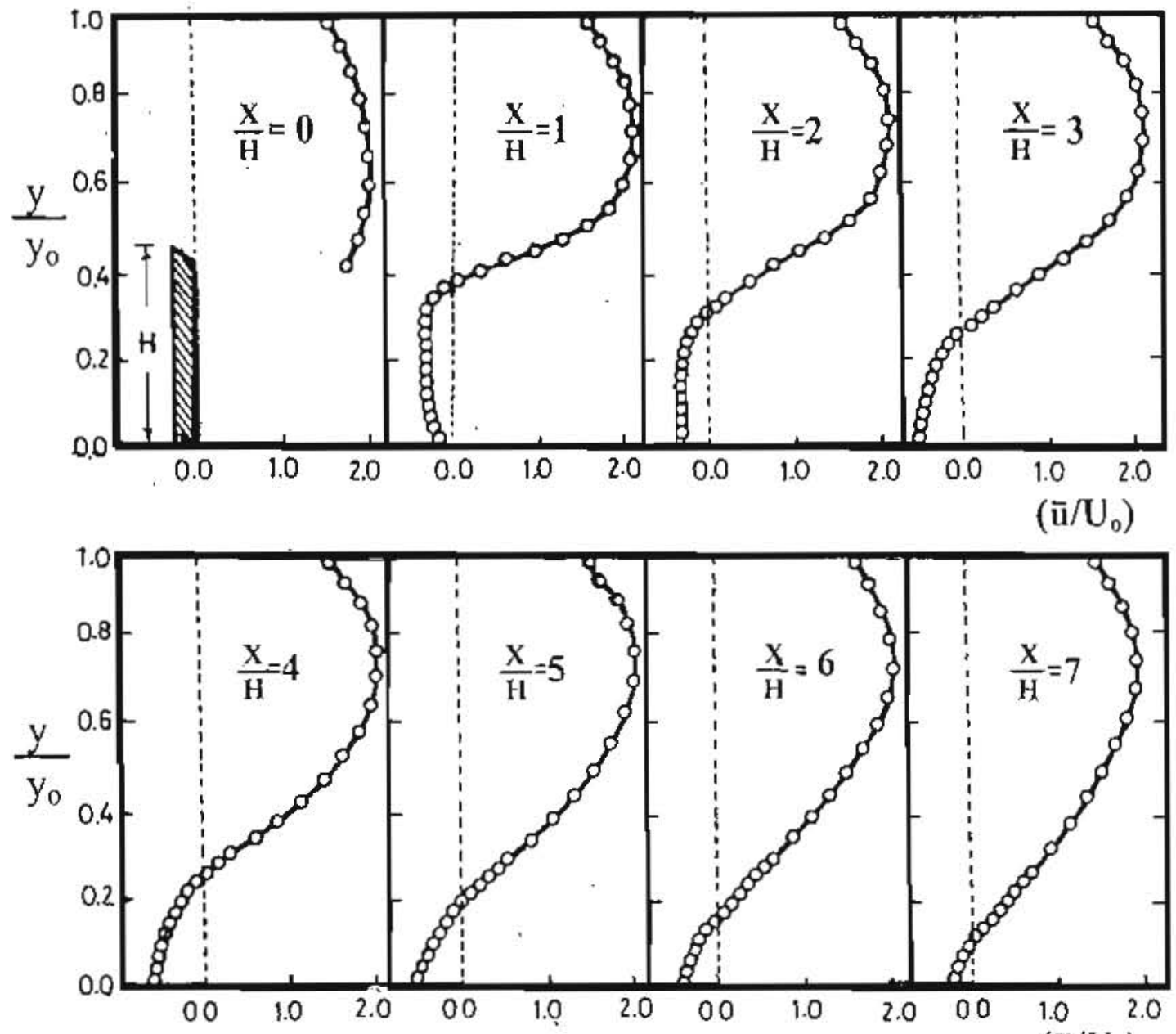

$\left(\overline{\mathrm{u}} / \mathrm{U}_{0}\right)$

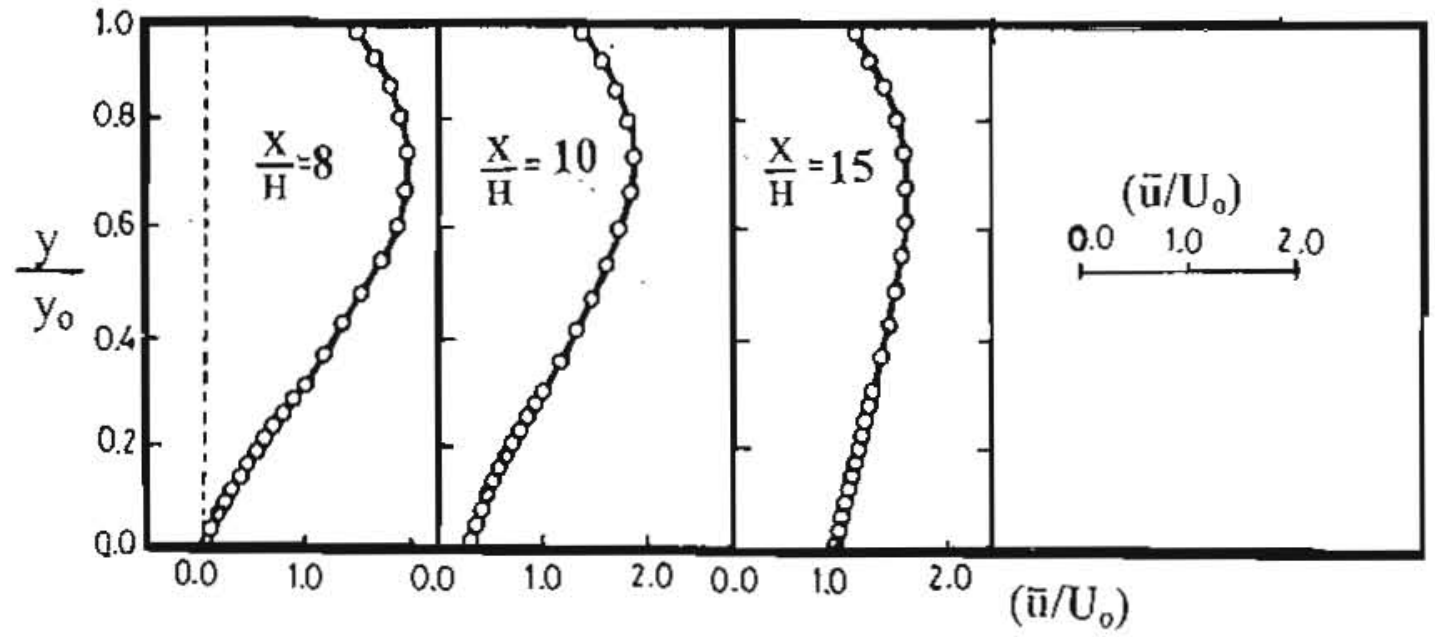

Fig. (5) Variation of streamwise mean velocity $\bar{u} / U_{0}$ with $y / y_{0}$ downstream the rectangular sharp crested weir. 


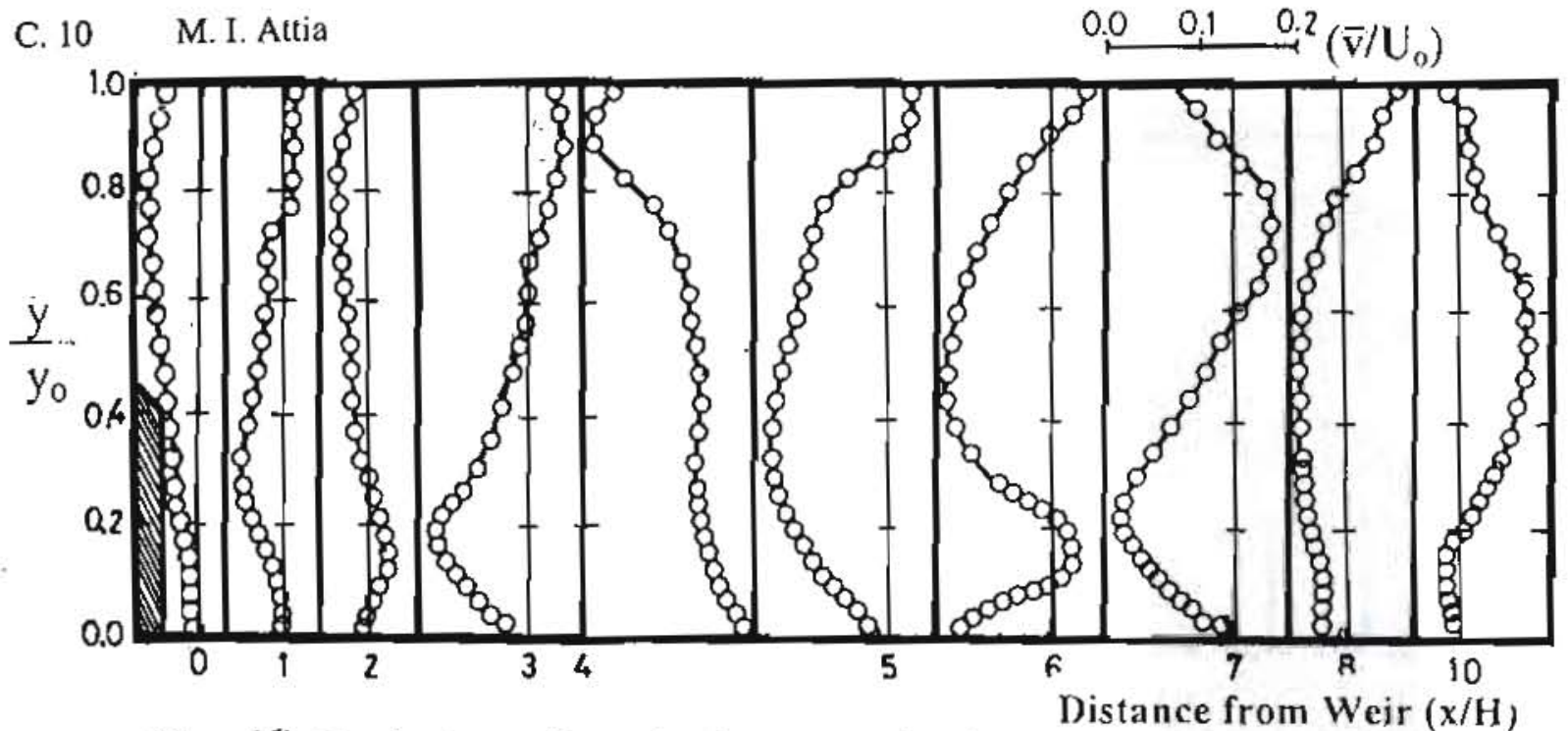

Fig. (6) Variation of vertical mean velocity $\bar{v} / U_{0}$ with $y / y_{0}$ downstream the rectangular sharp crested wcir
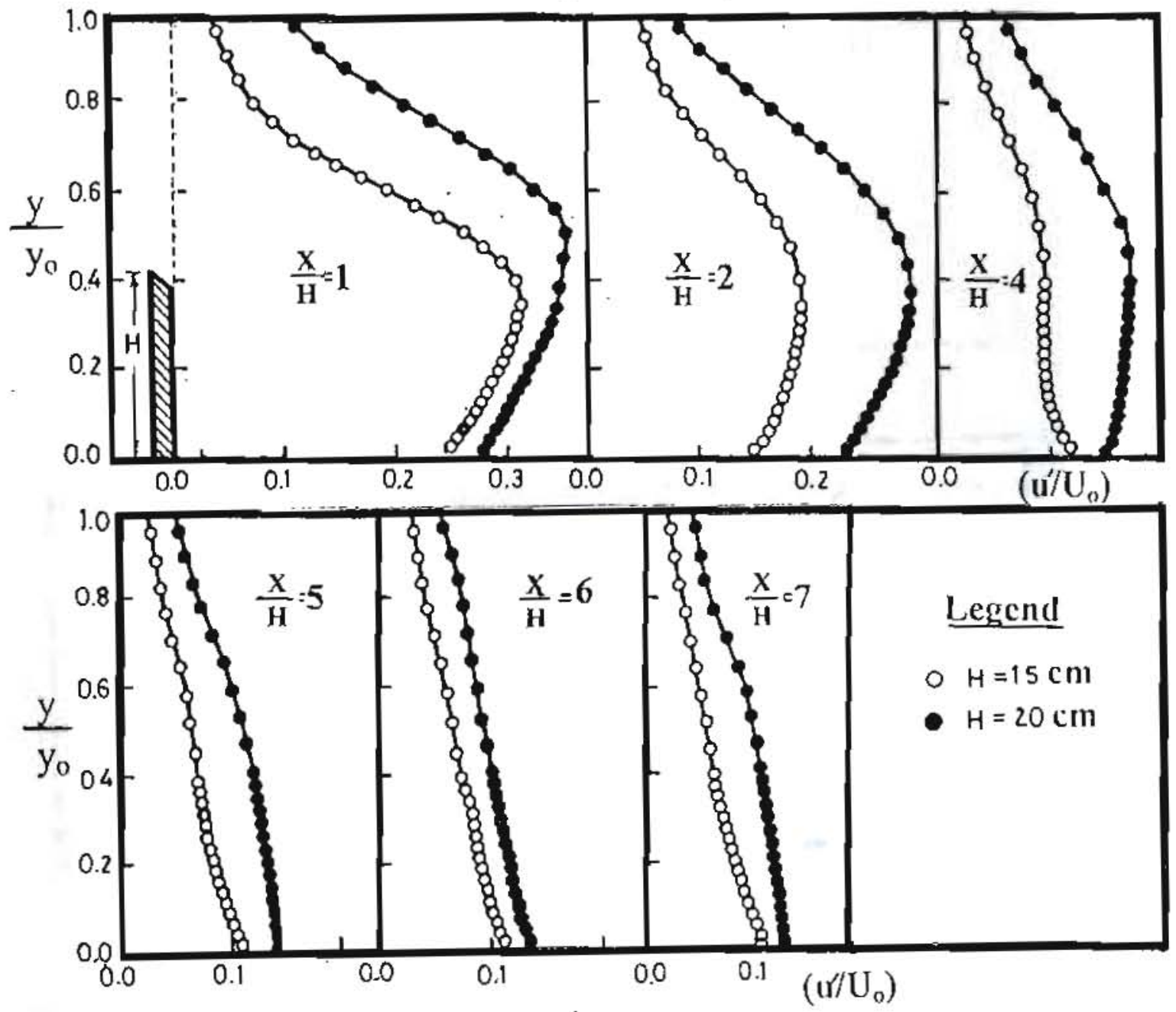

Fig. (7) Variation of stream wise turbulence intensity $\left(u^{\prime} / \mathrm{U}_{0}\right)$ with $y / y_{0}$ behind the sharp crested weir for different heights. 
Mansoura Engineering Joumal, (MEJ), Vol. 33, No. 3, September 2008.
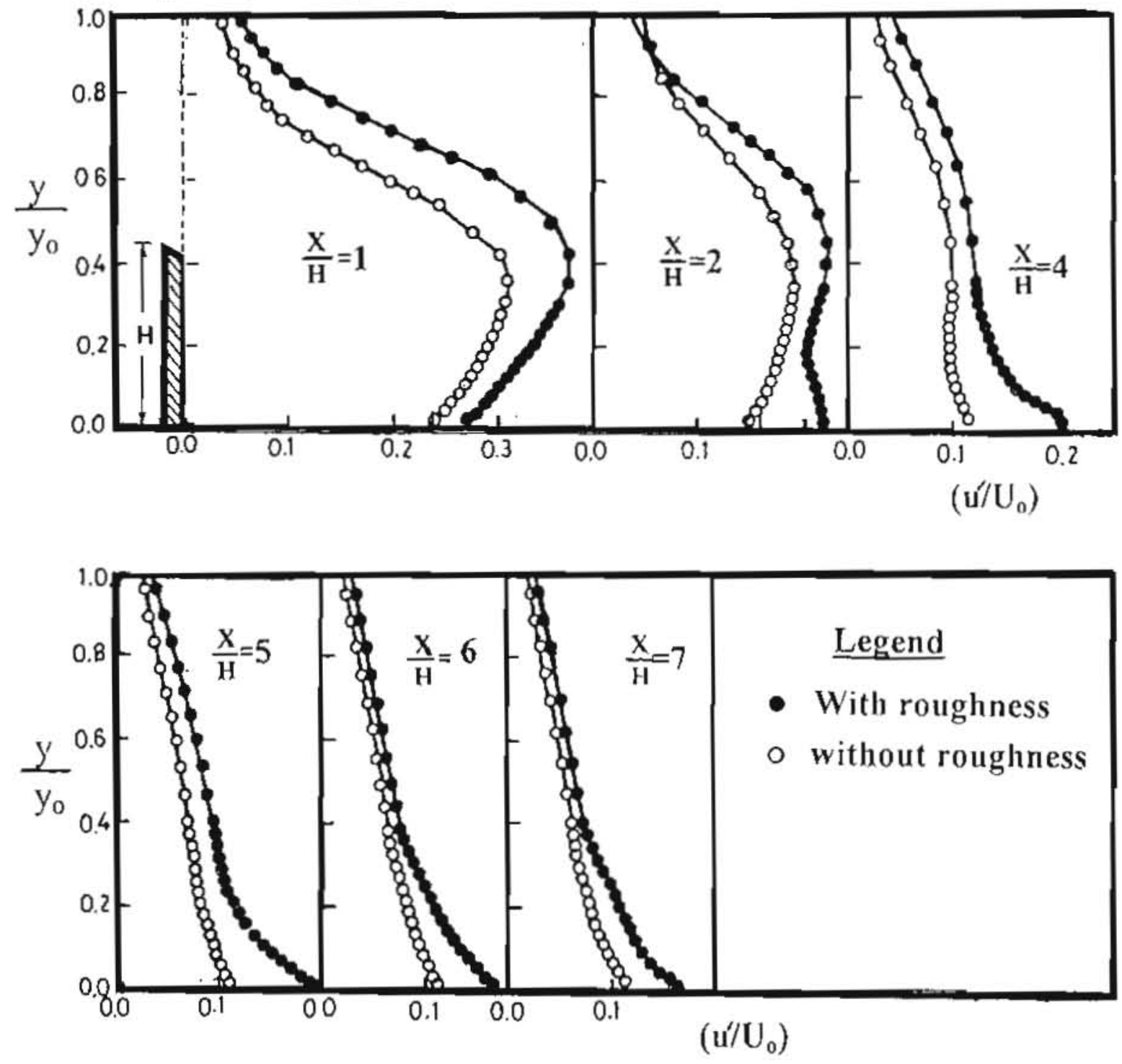

Fig. (8) Variation of streamwise turbulence intensity ( $\mathrm{u}^{\prime} / \mathrm{U}_{0}$ ) witl $y / y_{0}$ behind the rough sharp crested weir. 